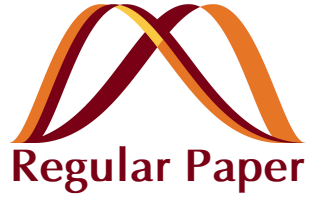

\section{Optimization of the Profiles in MeV Implanted Silicon Through the Modification of Electronic Stopping Power}

\author{
Won-Chae Jung ${ }^{\dagger}$ \\ Department of Electronic Engineering, Kyonggi University, Suwon 440-760, Korea
}

Received November 7, 2012; Accepted February 19, 2013

\begin{abstract}
The elements B, P and As can each be implanted in silicon; for the fabrication of integrated semiconductor devices and the wells in CMOS (complementary metal oxide semiconductor). The implanted range due to different implanted species calculated using TRIM (Transport of Ions in Matter) simulation results was considered. The profiles of implanted samples could be measured using SIMS (secondary ion mass spectrometry). In the comparison between the measured and simulated data, some deviations were shown in the profiles of MeV implanted silicon. The Moliere, $\mathrm{C}-\mathrm{Kr}$, and ZBL potentials were used for the range calculations, and the results showed almost no change in the MeV energy region. However, the calculations showed remarkably improved results through the modification of the electronic stopping power. The results also matched very well with SIMS data. The calculated tolerances of $R_{p}$ and $\Delta R_{p}$ between the modified $\mathrm{S}_{\mathrm{e}}$ of TRIM and SIMS data were remarkably better than the tolerances between the TRIM and SIMS data.
\end{abstract}

Keywords: B, P, As, Modification of $\mathrm{S}_{\mathrm{e}}$, MeV implantations, Secondary ion mass spectrometry, Computer simulation

\section{INTRODUCTION}

For the fabrication of CMOS devices, the retrograde wells of CMOS can be formed by using B, $\mathrm{P}$ and As ion implantations for $\mathrm{p}^{+}$and $\mathrm{n}^{+}$doping. For deep junctions, such impurities are implanted in silicon using an $\mathrm{MeV}$ implanter, and implanted wafers are annealed for electrical activation in $\mathrm{N}_{2}$ atmosphere. For the fabrication of power BICMOSFETs (bipolar complementary metal oxide semiconductor field effect transistors), accurate control of the depth profile is very important. Nowadays, SRIM (Stopping and Range of Ions in Matter) Monte Carlo simulations are widely used for the calculation of the implanted range in targets [1,2]. The physical effects of the slowing mechanism of implanted ions in silicon have also been examined extensively [1-10]. Experiments on implantations using $\mathrm{MeV}$ accelerators have been carried out for the fabrication of semiconductor devices, and the dynamics in implanted silicon have been analyzed using RBS

${ }^{\dagger}$ Author to whom all correspondence should be addressed:

E-mail: wcjung@kyonggi.ac.kr

Copyright @2013 KIEEME. All rights reserved.

This is an open-access article distributed under the terms of the Creative Commons Attribution Non-Commercial
License (http://creativecommons.org/licenses/by-nc/3.0) which permits unrestricted noncommercial use, distribution, and reproduction in any medium, provided the original work is properly cited.
(Rutherford Backscattering Spectrometry). The exact calculation of range in the $\mathrm{MeV}$ region is very important in semiconductor technology. However, some deviations in the ion range and defect formations between theoretical and experimental data have not yet been fully explained. TRIM (Transport of Ions in Matter) [1,2] was used as a Monte Carlo simulation tool for onedimensional profiles in various targets. Four moments were used for moment calculations in the one-dimensional (1D) vertical direction. The first moment $\mathrm{R}_{\mathrm{p}}$ (projected average range) is the average range under normal implantation, the second moment $\Delta \mathrm{R}_{\mathrm{p}}$ (standard deviation) represents the width of profile, the third moment $\gamma$ (skewness) indicates the asymmetry of the profile, and the fourth moment $\beta$ (kurtosis) represents the extent of the profile sharpness in the peak-concentration area. SRIM2013 output data was compared with measured SIMS data. [17]. For the moment calculations in the vertical direction, the following equations were used:

$$
C(x)=C_{\text {dose }} \cdot f(x)
$$

where $\mathrm{C}_{\text {dose }}$ is an implanted dose, $\mathrm{C}(\mathrm{x})$ is concentration as a func- 
tion of the depth $\mathbf{x}$, and $f(x)$ is a normalized distribution function. The four moments are defined as:

$$
\begin{aligned}
& R_{p}=\int_{0}^{\infty} x \cdot f(x) \\
& \Delta R_{p}=\sqrt{\int_{0}^{\infty}\left(x-R_{p}\right)^{2} \cdot f(x) d x} \\
& \gamma=\frac{\int_{0}^{\infty}\left(x-R_{p}\right)^{3} \cdot f(x) d x}{\Delta R_{p}{ }^{3}} \\
& \beta=\frac{\int_{0}^{\infty}\left(x-R_{p}\right)^{4} \cdot f(x) d x}{\Delta R_{p}{ }^{4}}
\end{aligned}
$$

A Gaussian profile has only two parameters due to symmetry, and can be expressed as:

$$
C(x)=\frac{C_{\text {dose }}}{\sqrt{2 \pi} \Delta R_{p}} \exp \left(-\frac{1}{2}\left[\frac{x-R_{p}}{\Delta R_{p}}\right]^{2}\right)
$$

The projected average range $\left(R_{p}\right)$ of a Gaussian profile is located near the peak concentration.

A Gaussian profile is generally not adequate to express asymmetry profiles for B-implanted, and P-implanted silicon. In the high energy region, the equations of electronic energy loss straggling and nuclear energy loss straggling can also be expressed as the equations below.

$$
\begin{aligned}
& Q_{n}=\frac{4 \pi Z_{1} Z_{2} e^{4} N}{\left(1+\frac{M_{2}}{M_{1}}\right)^{2}} W(\varepsilon) \\
& W(\varepsilon)=\frac{1}{1+1.646 \varepsilon^{-1.0494}+0.0492 \varepsilon^{-1.6991}}
\end{aligned}
$$

In the equation $8, \varepsilon$ is the reduced energy as described in equation 9 .

$$
\varepsilon=E \frac{a M_{2}}{Z_{1} Z_{2} e^{2}\left(M_{1}+M_{2}\right)}
$$

For low (L. E.) and high energy (H. E.) regions, $Q_{\mathrm{e}}$ can be expressed as equation 10 .

$$
Q_{e}=\operatorname{Min}\left\{\begin{array}{l}
3.2[\mathrm{keV}]^{-1} N\left(Z_{1}+Z_{2}\right)^{\frac{8}{3}} \frac{E[\mathrm{keV}]}{M_{1}} \rightarrow \text { L.E. } \\
4 \pi N Z_{1} Z_{2} e^{4} \rightarrow \text { H.E. }
\end{array}\right.
$$

Where $\mathrm{W}(\varepsilon)$ is the analytical ZBL function, $\mathrm{Z}_{1}, \mathrm{Z}_{2}, \mathrm{M}_{1}, \mathrm{M}_{2}$, and $\mathrm{E}$ are the incident ion number, target atom number, incident ion mass, target atom mass, and incident ion energy, $\mathrm{N}$ is the target atomic density, e is the elemental charge, and a is the screen length, respectively. $\mathrm{Q}_{\mathrm{e}}$ is the electronic energy loss straggling. This straggling $Q_{e}$ is dominant, when a light element implanted silicon. In the experiments, only boron implant corresponded to the domination of $\mathrm{Q}_{\mathrm{e}}$ in silicon. Heavy elements in implanted

\begin{tabular}{cccc} 
Table 1 Constants in the screening & Munctions for various potentials \\
\hline \hline & Moliere & Kr-C & ZBL \\
\hline \hline $\mathrm{n}$ & 3 & 3 & 4 \\
\hline $\mathrm{c}_{1}$ & 0.35 & 0.190945 & 0.028171 \\
\hline $\mathrm{c}_{2}$ & 0.55 & 0.473674 & 0.28022 \\
\hline $\mathrm{c}_{3}$ & 0.1 & 0.335381 & 0.50986 \\
\hline $\mathrm{c}_{4}$ & 0 & 0 & 0.18175 \\
\hline $\mathrm{d}_{1}$ & 0.3 & 0.278544 & 0.20162 \\
\hline $\mathrm{d}_{2}$ & 1.2 & 0.637174 & 0.40290 \\
\hline $\mathrm{d}_{3}$ & 6.0 & 1.919249 & 0.94229 \\
\hline $\mathrm{d}_{4}$ & 0 & 0 & 3.1998 \\
\hline
\end{tabular}

silicon caused the ion distribution mainly through nuclear stopping power. The stopping power $S_{e}$ and $S_{n}$ are defined as average energy. In an individual process, there is a statistically fluctuating energy loss in the ion implantation. In the high energy region, the equations of electronic energy loss straggling $Q_{e}$ and nuclear energy loss straggling $\mathrm{Q}_{\mathrm{n}}$ can also be expressed in equations 7 and 10 , respectively.

The screen length can be expressed by equation 11 .

$$
a=\left(\frac{9 \pi^{2}}{128}\right)^{\frac{1}{3}} \frac{a_{0}}{\left(Z_{1}^{x}+Z_{2}^{x}\right)^{y}}
$$

where $\alpha_{0}$ is Bohr's radius.

In the high energy region of the TRIM program, the standard deviations in the projected direction can be expressed as:

$$
\left(\Delta R_{p}\right)^{2}=\int_{0}^{E_{0}} \frac{Q_{e}+Q_{n}}{\left(S_{e}+S_{n}\right)^{3}} d E=\int_{0}^{E_{0}} \frac{Q_{t}}{\left(S_{t}\right)^{3}} d E
$$

Three different models, the Firsov-, Lindhard et al.-, and ZBL (Ziegler, Biersack, Littmark) models [16,17], can be expressed as $x=1 / 2$, and $y=2 / 3$ for the Firsov-model, $x=2 / 3$, and $y=1 / 2$ for the Lindhard et al.-model, and $\mathrm{x}=0.23$, and $\mathrm{y}=1.0$ for the ZBL-model. The screened Coulomb'-potentials are expressed in equations 13, and 14, and in Table 1.

$$
\begin{aligned}
& V(r)=\frac{Z_{1} Z_{2} e^{2}}{r} \phi\left(\frac{r}{a}\right)=\frac{Z_{1} Z_{2} e^{2}}{a(r / a)} \phi\left(\frac{r}{a}\right)=\frac{C}{(r / a)} \phi\left(\frac{r}{a}\right) \\
& \phi\left(\frac{r}{a}\right)=\sum_{i=1}^{n} c_{i} \exp \left(-d_{i} \frac{r}{a}\right), \quad \sum_{i=1}^{n} c_{i}=\phi(0)=1
\end{aligned}
$$

In $\mathrm{MeV}$ regions $(\mathrm{r} / \mathrm{a}<1)$, the screening effects of various interatomic potentials are almost the same.

Therefore, the range parameters $R_{p}$ and $\Delta R_{p}$ are also almost the same value in the three potentials. Nevertheless, the range values after computer simulations were always shown with $\mathrm{R}_{\mathrm{ZBL}}>\mathrm{R}_{\text {Moliere }}>\mathrm{R}_{\mathrm{Kr}-\mathrm{C}}$ with very small differences in values. There were no differences in the range values between $\mathrm{ZBL}$ and Moliere potentials, but the range values of the $\mathrm{Kr}-\mathrm{C}$ potential were somewhat smaller.

\section{EXPERIMENTS OF B, P AND As IMPLANTATIONS}

P-type silicon wafers-, doped with (100) boron and $\rho$ values of 4-5 $\Omega \mathrm{cm}$-, were used. The concentration of the silicon substrate was about $3 \times 10^{15} \mathrm{~cm}^{-3}$. For the $\mathrm{p}$ and n-type doping of retrograde well formations for CMOS fabrication, B, P and As ions were implanted in silicon wafers. Implantations into the silicon sub- 


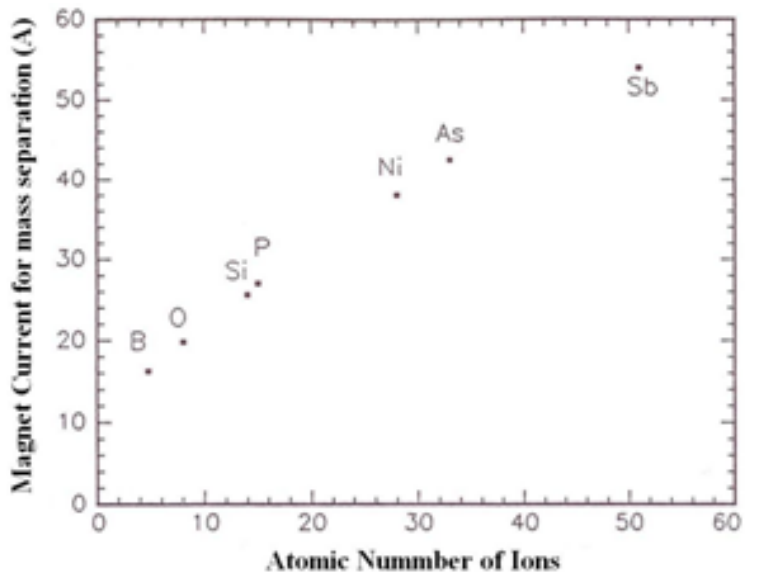

Fig. 1. Magnet current for the mass separation of various ions in the first magnet.

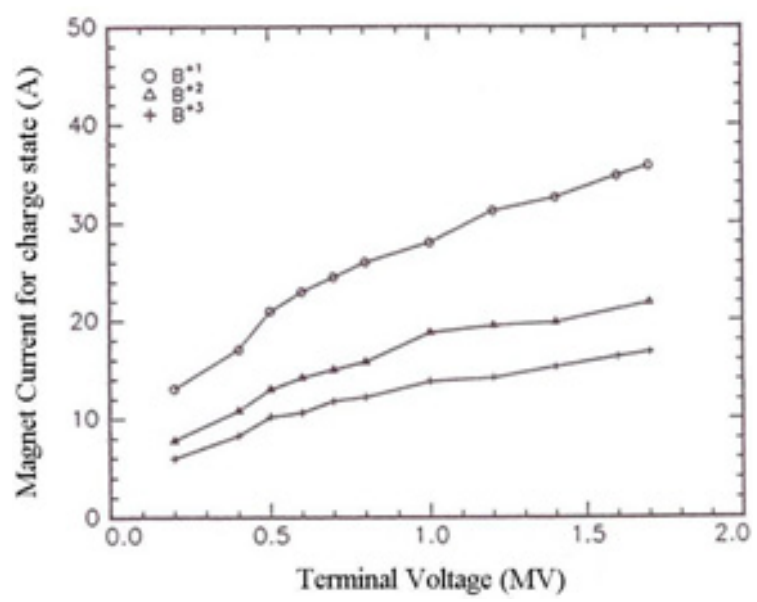

Fig. 2. Magnet current for the charge separation of boron ions in the second magnet.

strate with different doses were carried out using a tandem ion implanter.

Before the implantation, the accelerated energies were calculated from the injection energy, charge state, and terminal voltage. The final implanted energy can be calculated using equation 15 :

$$
\mathrm{E}_{\mathrm{tot}}=\mathrm{q}\left[\mathrm{V}_{\mathrm{inj}}+(1+\mathrm{n}) \mathrm{V}_{\mathrm{T}}\right]
$$

where $V_{\text {in }}, n$, and $V_{T}$ are the pre-acceleration voltage $(60 \mathrm{~V})$, charge state of ions, and terminal voltage, respectively. A cesium $\left(\mathrm{Cs}^{+}\right)$ion sputtering source was used for the extraction of negative ions. The negative ions are attracted with the terminal voltage and the accelerated ions are collided with the $\mathrm{N}_{2}$-stripped gas in the middle zone. The negative ions can be changed into positive ions. After that, the positive ions are repelled again and focused through the electrical field lines with three quadrupoles.

The vacuum inside the accelerator was $1 \times 10^{-7}$ torr, and the space between the electrode and the tube was filled $\mathrm{SF}_{6}$ gas for electrical isolation. This accelerator has two magnets for the implantation and RBS. The first magnet was used for the mass separation in the injector parts and the second magnet was used for the separation of charge states in the front part of the end sta-

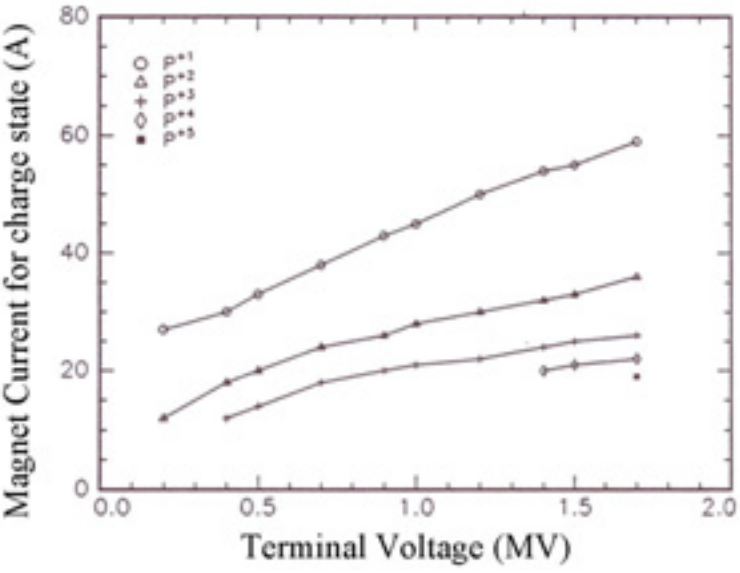

Fig. 3. Magnet current for the charge separation of phosphorus ions in the second magnet.

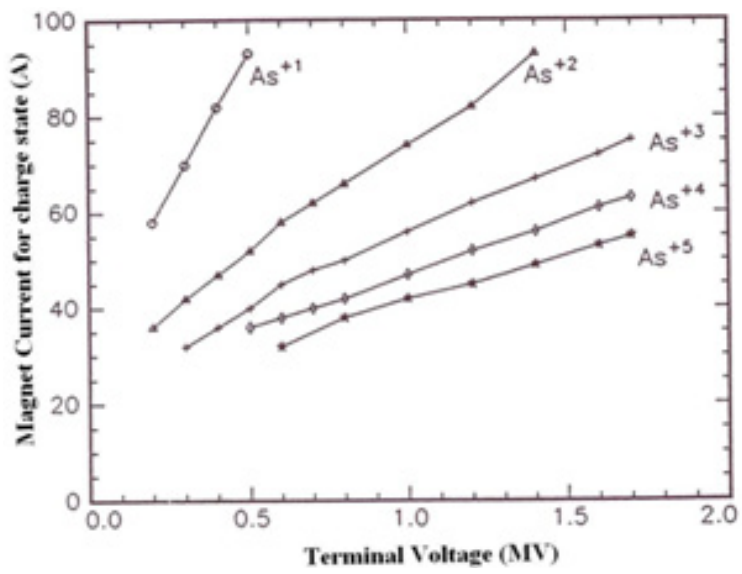

Fig. 4. Magnet current for the charge separation of arsenic ions in the second magnet.

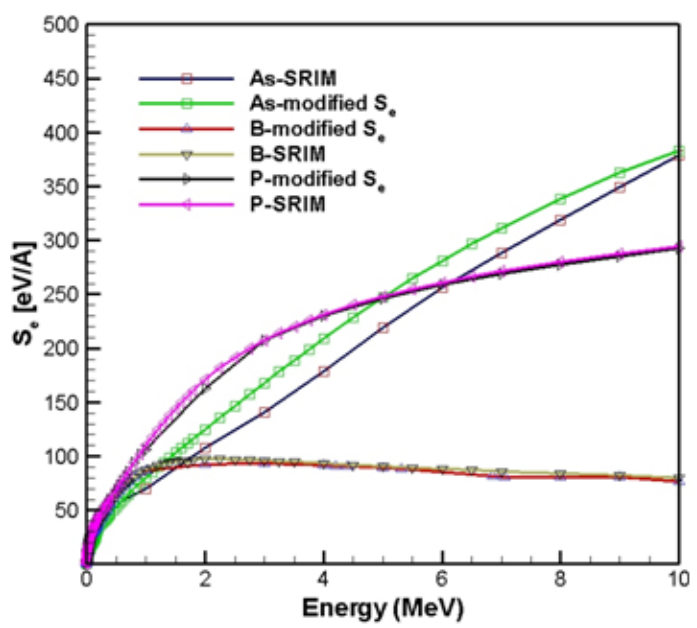

Fig. 5. Comparison of electronic stopping power between SRIM (2013) and modified curve.

tion. The magnet current for the mass separation of various ions in the first magnet is shown in Fig. 1. The magnet currents for the charge separation of boron, phosphorus and arsenic ions in the second magnet are shown in Figs. 2, 3 and 4, respectively. 
The modified electronic stopping powers of SRIM and SRIM2013 are shown in Fig. 5.

Boron, phosphorus, and arsenic ions implanted in silicon show somewhat shallower depth in the SIMS data. This means that the electronic stopping powers of SRIM are overestimated in implanted silicon. Based on this, the electronic stopping powers of SRIM are modified and optimized by comparing with measured SIMS data.

The fitted curves of boron-implanted silicon are obtained by the modification of the electronic stopping power of SRIM as follows;

$$
\begin{aligned}
& \mathrm{S}_{\mathrm{e}}(\mathrm{E})=24 \mathrm{E}^{0.2}-0.0085 \mathrm{E} \text { for } 1 \sim 6 \mathrm{MeV} \\
& \mathrm{S}_{e}(\mathrm{E})=24 \mathrm{E}^{0.2}-0.008 \mathrm{E} \text { for } 7 \sim 8 \mathrm{MeV} \\
& \mathrm{S}_{e}(\mathrm{E})=24 \mathrm{E}^{0.2}-0.0075 \mathrm{E} \text { for } 9 \sim 10 \mathrm{MeV}
\end{aligned}
$$

where the energy $\mathrm{E}$ and electronic stopping power $\mathrm{S}_{\mathrm{e}}$ are in units of $1,000 \mathrm{keV}$ and $\mathrm{eV} / \AA$, respectively.

The fitted equations could not be expressed as only one equation because of the broad energy regions from 1 to $10 \mathrm{MeV}$.

The fitted curves for phosphorus-implanted silicon are obtained by the modification of the electronic stopping power of SRIM in equations (19-21):

$$
\begin{aligned}
& S_{e}(E)=1.4 E^{0.64}-0.009 E \text { for } 1 \sim 5 \mathrm{MeV} \\
& S_{e}(E)=1.4 E^{0.64}-0.016 E \text { for } 6 \sim 7 \mathrm{MeV} \\
& S_{e}(E)=1.4 E^{0.64}-0.017 E \text { for } 8 \sim 10 \mathrm{MeV}
\end{aligned}
$$

The fitted curves in arsenic-implanted silicon are obtained by the modification of the electronic stopping power of SRIM in equations (22-25):

$$
\begin{aligned}
& S_{e}(E)=1.7 E^{0.5}+0.016 E \text { for } 1 \sim 3 \mathrm{MeV} \\
& S_{e}(E)=1.7 E^{0.5}+0.018 \text { for } 4 \mathrm{MeV} \\
& S_{e}(E)=1.7 E^{0.5}+0.02 \text { for } 5 \mathrm{MeV} \\
& S_{e}(E)=1.7 E^{0.5}+0.021 \text { for } 6 \sim 10 \mathrm{MeV}
\end{aligned}
$$

where the energy $\mathrm{E}$ and electronic stopping power $\mathrm{S}_{\mathrm{e}}$ mean the unit of $1,000 \mathrm{keV}$ and $\mathrm{eV} / \AA$, respectively.

The electronic stopping power $S_{e}$ and nuclear stopping power $\mathrm{S}_{\mathrm{n}}$ in B-implanted silicon are shown in Fig. 6.

The value of $\mathrm{S}_{\mathrm{e}}$ was $87.62 \mathrm{eV} / \AA$ at $1 \mathrm{MeV}$, and $97.24 \mathrm{eV} / \AA$ at a peak point at $2.25 \mathrm{MeV}$. On the other side, $\mathrm{S}_{\mathrm{n}}$ showed a very low value of $0.5814 \mathrm{eV} / \AA$ at $1 \mathrm{MeV}$, and continuously decreased gradually between 1 and $3 \mathrm{MeV}$. This means that $S_{n}$ can actually be disregarded in MeV. The solid-dot-dot line in the figure shows a modified $\mathrm{S}_{\mathrm{e}}$ line, which has somewhat values than those obtained from TRIM data. The range data showed better results than the TRIM data using a modification of $S_{e}$ in B-implanted silicon. The TRIM data was obtained using SRIM 2013 [17]. For good fitting with SIMS data the somewhat lower values of $\mathrm{S}_{\mathrm{e}}$ than the $\mathrm{S}_{\mathrm{e}}$ of SRIM 2013 are corrected for the modifications of $\mathrm{S}_{\mathrm{e}}$ in $\mathrm{B}$,

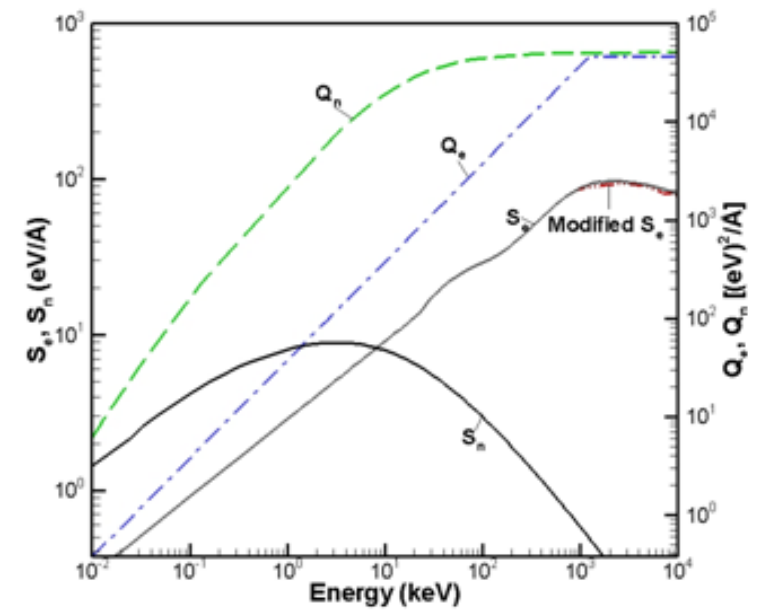

Fig. 6. $S_{n}, S_{e}$ of TRIM and modified TRIM with $Q_{e}$ and $Q_{n}$ in B implanted silicon.

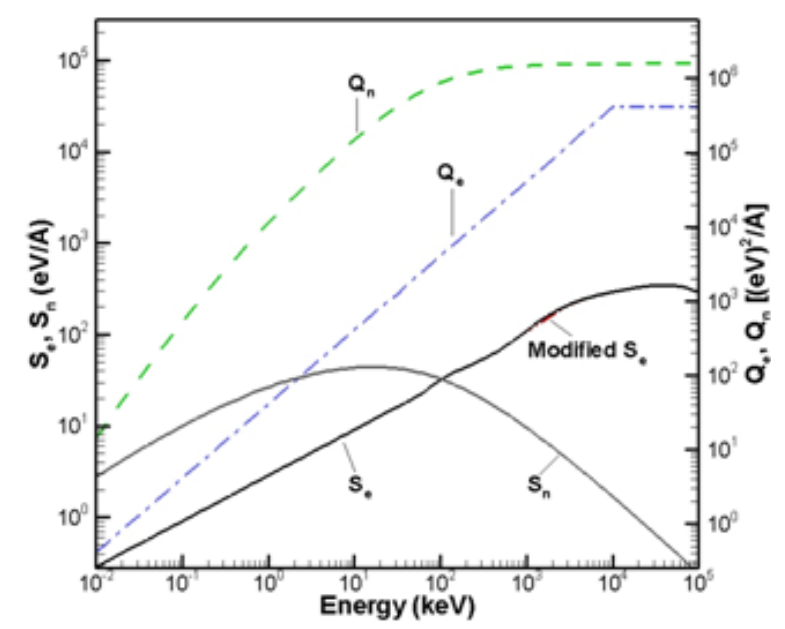

Fig. 7. $S_{n}, S_{e}$ of TRIM and modified TRIM with $Q_{e}$ and $Q_{n}$ in P implanted silicon.

$\mathrm{P}$, and As in implanted silicon. The measured SIMS data of B, P, and As in implanted silicon were slightly higher than the TRIM simulated data. The $S_{n}$ and $S_{e}$ data can be used for the range calculation for implanted profiles. For this reason, the nuclear stopping power $S_{n}$ of the ZBL model [17] was substituted with the Moliere and $\mathrm{Kr}-\mathrm{C}$ models [16], and the simulated range data were very similar. The range data showed the best results using the ZBL model in comparison with the SIMS data. The $S_{n}$ value of $\mathrm{B}$ in $\mathrm{MeV}$ implanted silicon could be disregarded due to very low values ranging from $0.5814 \mathrm{eV} / \AA$ at $1 \mathrm{MeV}$ to $0.08734 \mathrm{eV} / \AA$ at $10 \mathrm{MeV}$ in TRIM data. The electronic stopping power $S_{e}$ plays an important role in the $\mathrm{MeV}$ region, and can be modified from the original TRIM value. The changed curves are shown in Fig. 5 and 6.

The range data are also showed better results than TRIM data using a modification of $\mathrm{S}_{\mathrm{e}}$ in P-implanted silicon. The value of TRIM $\mathrm{S}_{\mathrm{e}}$ was $110 \mathrm{eV} / \AA$ at $1 \mathrm{MeV}$, and $294.5 \mathrm{eV} / \AA$ at $10 \mathrm{MeV}$, with a continuously increasing sloped line, as shown in Fig. 7 . The peak $S_{n}$ value of P implanted in silicon was $44.2 \mathrm{eV} / \AA$ at $15 \mathrm{keV}$. The value $S_{n}$ of $\mathrm{P}$ was relatively low, ranging from $9.549 \mathrm{eV} / \AA$ at 1 $\mathrm{MeV}$ to $1.688 \mathrm{eV} / \AA$ at $10 \mathrm{MeV}$ in TRIM data. The electronic stopping power $\mathrm{S}_{\mathrm{e}}$ can be modified from the original TRIM value. The changed curves in P-implanted silicon are shown in Figs. 5 and 7. 


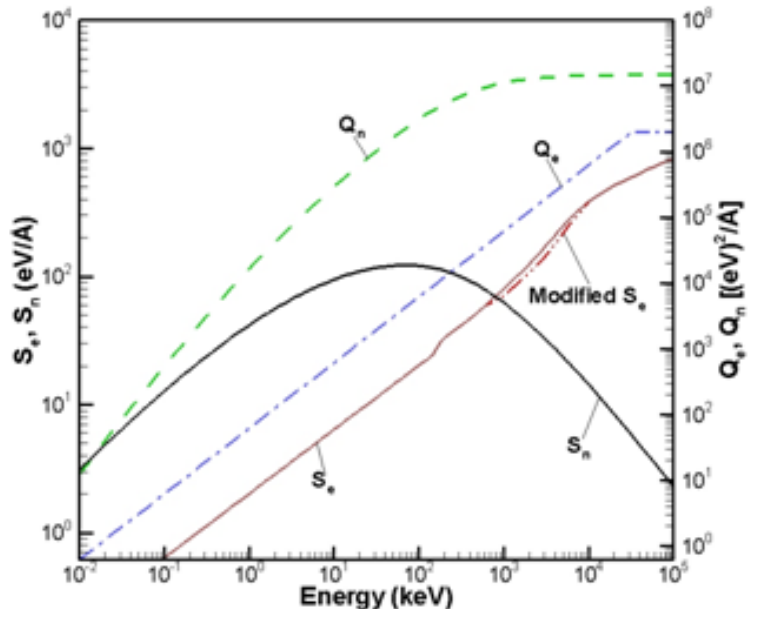

Fig. 8. $S_{n}, S_{e}$ of TRIM and modified TRIM with $Q_{e}$ and $Q_{n}$ in As implanted silicon.

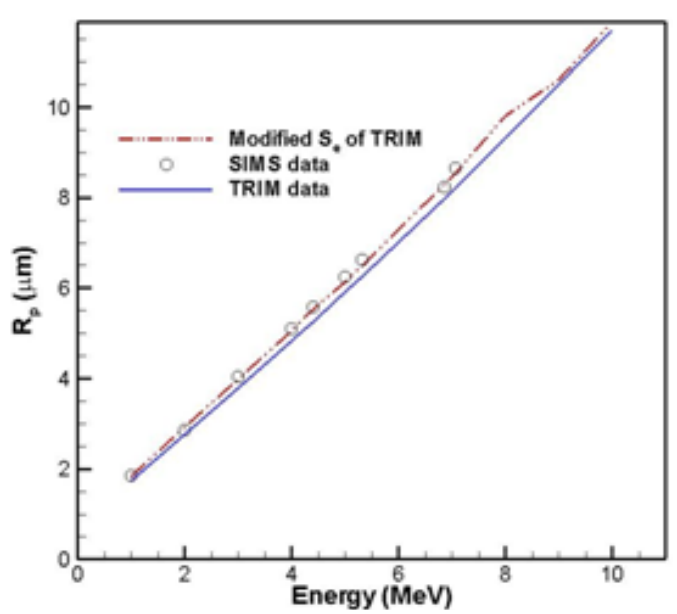

Fig. 9. Comparison of B projected average ranges with TRIM, SIMS and modified $\mathrm{S}_{\mathrm{e}}$ of TRIM.

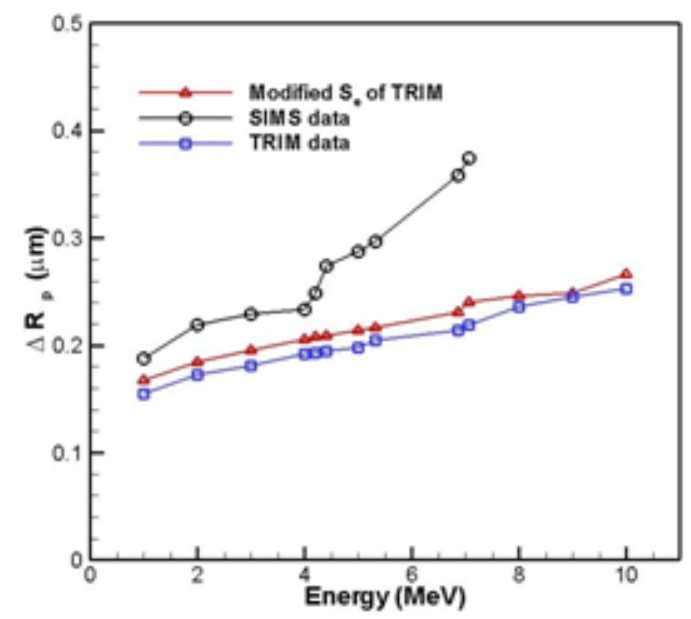

Fig. 10. Comparison of B projected standard deviations with TRIM, SIMS and modified $\mathrm{S}_{\mathrm{e}}$ of TRIM.

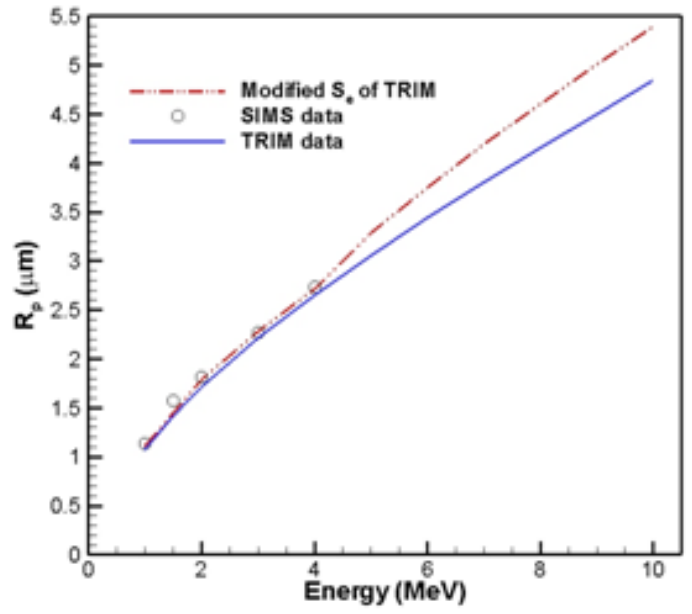

Fig. 11. Comparison of P projected average ranges with TRIM, SIMS and modified $\mathrm{S}_{\mathrm{e}}$ of TRIM.

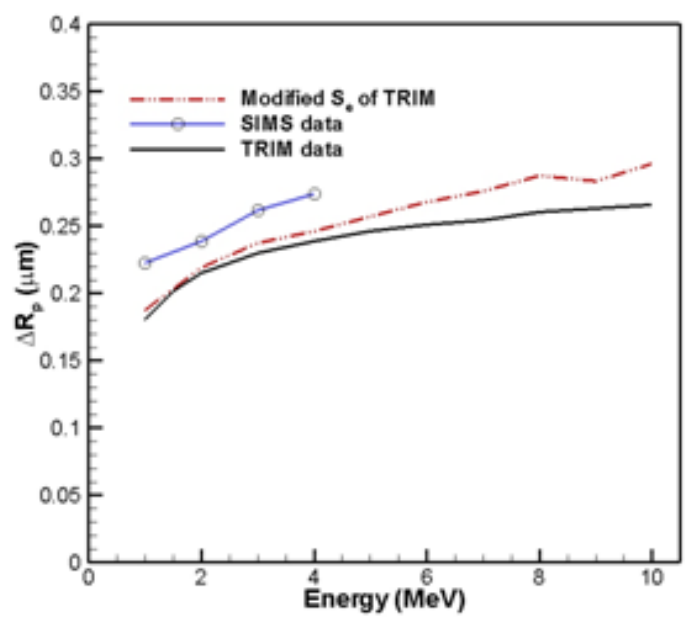

Fig. 12. Comparison of P projected standard deviations with TRIM, SIMS and modified $\mathrm{S}_{\mathrm{e}}$ of TRIM .

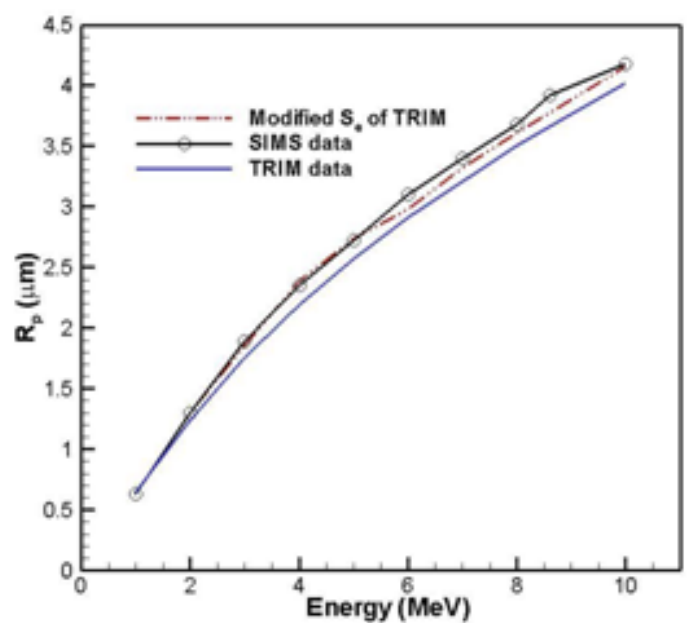

Fig. 13. Comparison of As projected average ranges with TRIM, SIMS and modified $\mathrm{S}_{\mathrm{e}}$ of TRIM . 


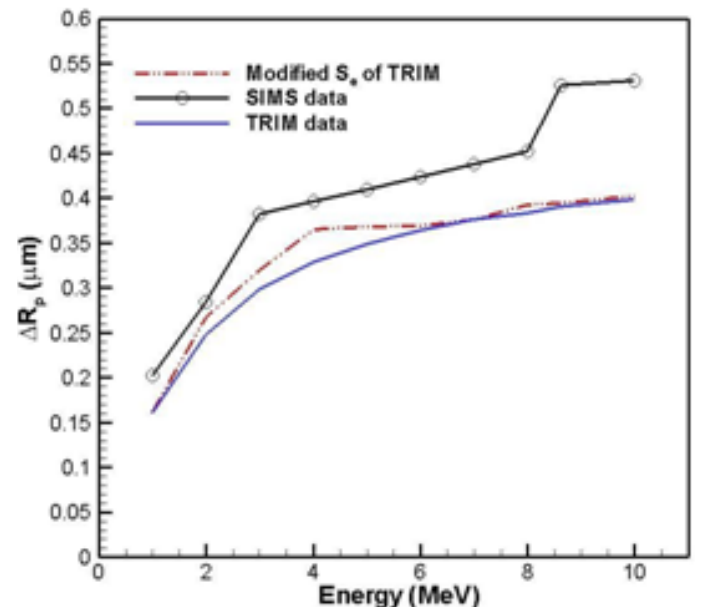

Fig. 14. Comparison of As projected standard deviations with TRIM, SIMS and modified $\mathrm{S}_{\mathrm{e}}$ of TRIM.

(a) Laterat

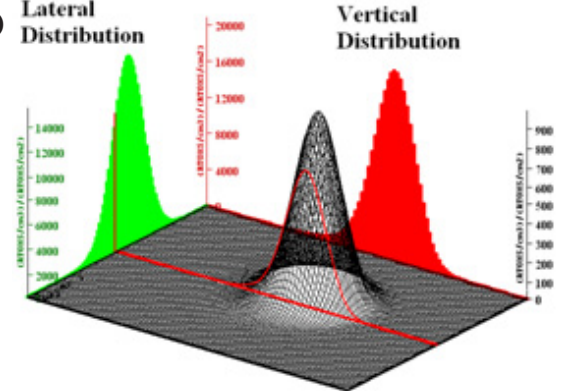

(b)

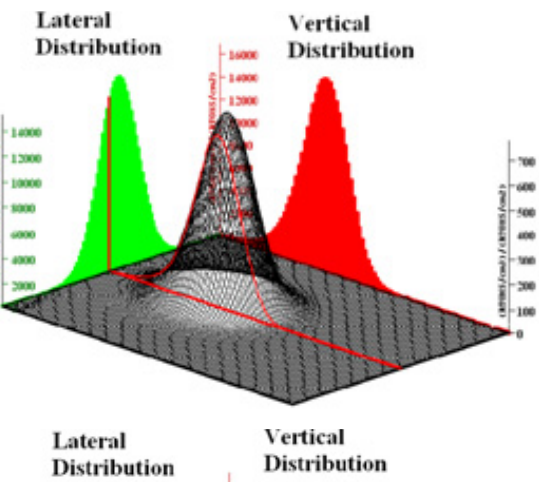

(c)

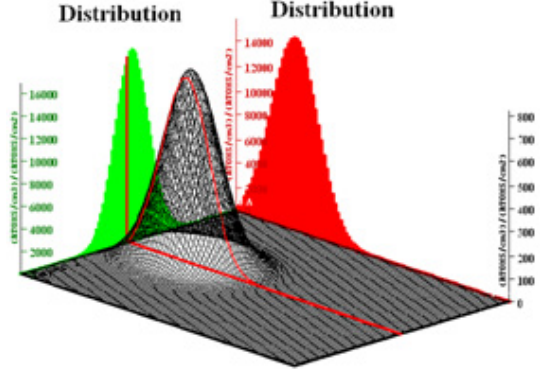

Fig. 15. Three-dimensional profiles in B, $\mathrm{P}$ and As implanted silicon using $1 \mathrm{MeV}$. (a) Boron profiles, (b) phosphorus profiles, and (c) Arsenic profiles.

The electronic stopping power $\mathrm{S}_{\mathrm{e}}$ of TRIM is somewhat overestimated in the $\mathrm{MeV}$ region.

The range data showed better results than the TRIM data using a modification of $\mathrm{S}_{\mathrm{e}}$ in As-implanted silicon. The electronic stopping power $\mathrm{S}_{\mathrm{e}}$ can be modified from the original TRIM value.

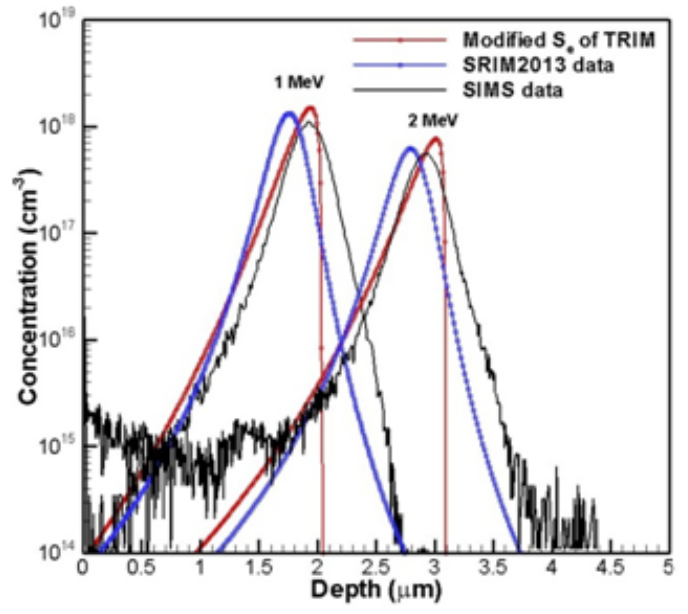

Fig. 16. Profiles from SIMS, SRIM and modified $\mathrm{S}_{\mathrm{e}}$ of TRIM in B implanted silicon using 1 and $2 \mathrm{MeV}$.

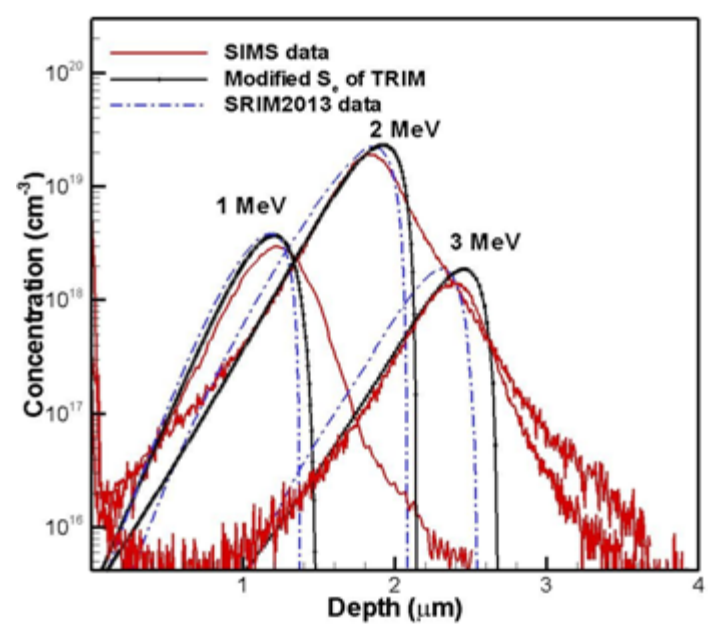

Fig. 17. Profiles from SIMS, SRIM and modified $\mathrm{S}_{\mathrm{e}}$ of TRIM in P implanted silicon using 1, 2 and $3 \mathrm{MeV}$.

The changed curves are represented as solid-dot-dot line for Asimplanted silicon in Figs. 5 and 8. The electronic stopping power $\mathrm{S}_{\mathrm{e}}$ is remarkably overestimated in the $\mathrm{MeV}$ region. The value of TRIM $\mathrm{S}_{\mathrm{e}}$, which was $80.26 \mathrm{eV} / \AA$ at $1 \mathrm{MeV}$, and $383.3 \mathrm{eV} / \AA$ at 10 $\mathrm{MeV}$, was a continuously increasing sloped line, as shown in Fig. 8. On the other hand, the $S_{n}$ value of As showed relatively low values ranging from $62.49 \mathrm{eV} / \AA$ at $1 \mathrm{MeV}$ to $14.36 \mathrm{eV} / \AA$ at $10 \mathrm{MeV}$ in TRIM data.

The simulated results of range calculations were shown from Fig. 9 to Fig. 14.

Three-dimensional B, P and As profiles of SRIM2013 are shown in Fig. 15. Every vertical length is $3 \mu \mathrm{m}$ in all three profiles. The shapes of the three profiles differ in regard to the positions and distributions due to the different ion masses and scattering angles.

The three-dimensional profiles in B, P, and As implanted silicon are shown in Fig. 15.

The positions of peak concentrations in the modified $\mathrm{S}_{\mathrm{e}}$ TRIM data are remakably improved in comparision with the SIMS and SRIM data in Figs. 16, 17, and 18.

The percentage of the tolerance between the experimental and simulated data can be expressed by equation 26 : 


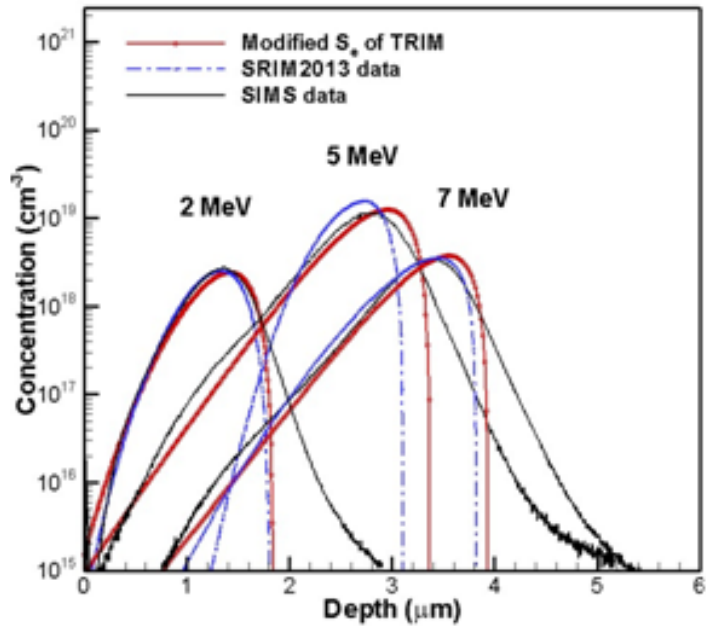

Fig. 18. Profiles from SIMS, SRIM and modified $\mathrm{S}_{\mathrm{e}}$ of TRIM in As implanted silicon using 2, 5 and $7 \mathrm{MeV}$.

$$
F=\left|1-\frac{\mathrm{R}_{\mathrm{p}} \text { or } \Delta \mathrm{R}_{\mathrm{p}}\left(\text { TRIM or modified } \mathrm{S}_{\mathrm{e}} \text { of TRIM }\right)}{\mathrm{R}_{\mathrm{p}} \text { or } \Delta \mathrm{R}_{\mathrm{p}}(\mathrm{SIMS})}\right| \times 100 \%
$$

The calculated tolerances of $R_{p}$ and $\Delta R_{p}$ between TRIM and SIMS data in B-implanted silicon from 1 to $10 \mathrm{MeV}$ were $5.53 \%$ and $20.5 \%$, respectively. The calculated tolerances of $R_{p}$ and $\Delta R_{p}$ between the modified $\mathrm{S}_{\mathrm{e}}$ of TRIM and SIMS data were remarkably improved by $1.98 \%$ and $15.8 \%$, respectively.

The calculated tolerances of $\mathrm{R}_{\mathrm{p}}$ and $\Delta \mathrm{R}_{\mathrm{p}}$ between TRIM and SIMS data in P-implanted silicon from 1 to $5 \mathrm{MeV}$ were $3.77 \%$ and $16.8 \%$, respectively. Those $R_{p}$ and $\Delta R_{p}$ between the modified $\mathrm{S}_{\mathrm{e}}$ of TRIM and SIMS data were remarkably improved by $1.44 \%$ and $13.8 \%$, respectively.

The calculated tolerances of $R_{p}$ and $\Delta R_{p}$ between TRIM and SIMS data in As-implanted silicon from 1 to $10 \mathrm{MeV}$ were $5.27 \%$ and $17.3 \%$, respectively. The tolerances between the modified $\mathrm{S}_{e}$ of TRIM and SIMS data were also remarkably improved by $2.64 \%$ and $15.3 \%$, respectively.

\section{CONCLUSIONS}

The range parameters, $R_{p}$ and $\Delta R_{p}$ of $B, P$ and As through the modifications of $S_{e}$ have been remarkably improved compared to results of SRIM2013, which has been continuously improved as a simulation tool since 1985 . The range parameters of $\mathrm{B}, \mathrm{P}$ and As obtained using ZBL potentials showed the best results among the various inter-atomic potentials (ZBL, Moliere, and $\mathrm{Kr}$ C). In experiments, B, P and As ions were implanted into silicon. The MeV implanted data showed small discrepancies between SIMS and simulated data of SRIM 2013, the newest version of the simulation tool. The SRIM 2013 data provided values of the electronic stopping power $\mathrm{S}_{\mathrm{e}}$ of $\mathrm{B}, \mathrm{P}$ and As ions in silicon that were somewhat overestimated. For this reason, the $S_{e}$ values of $\mathrm{B}, \mathrm{P}$, and As ions were modified to be slightly smaller, and the results of the calculation were optimized through modification of $\mathrm{S}_{\mathrm{e}}$. In the $\mathrm{MeV}$ regions, the electronic stopping power plays a dominant role in comparison with nuclear stopping powers. The fitted $S_{e}$ equations could not be expressed as only one equation because of broad energy regions from 1 to $10 \mathrm{MeV}$ in $\mathrm{B}, \mathrm{P}$, and As-implanted silicon. In the $\mathrm{MeV}$, the electronic stopping power of $\mathrm{B}$ and $\mathrm{P}$ were proportional to $\mathrm{E}^{0.2}$ and $\mathrm{E}^{0.64}$ instead of $\mathrm{E}^{0.5}$, as expressed in the LSS stopping power model [11-15]. The electronic stopping powers of As were proportional to $\mathrm{E}^{0.5}$, as expected for the heavy ions. Through the modifications of $\mathrm{S}_{\mathrm{e}}$ in $\mathrm{B}, \mathrm{P}$ and Asimplanted silicon, the range calculations were optimized and the range parameters showed better results than those of SRIM2013. The calculated tolerances of $R_{p}$ and $\Delta R_{p}$ between the modified $S_{e}$ of TRIM and SIMS data were also remarkably better than the tolerances between the TRIM and SIMS data.

\section{ACKNOWLEDGMENT}

This work was supported by a research grant from Kyonggi University, 2010.

\section{REFERENCES}

[1] U. Littmark and J. F. Ziegler, Phys. Rev. 23, (1980) [DOI: http:// dx.doi.org/10.1103/PhysRevA.23.64].

[2] J. P. Biersack and J. F. Ziegler, "Ion Implantation Techniques", Springer-Verlag, Berlin, (1982) p. 281

[3] W. C. Jung, J. KEEME, 15, 289, (2002)

[4] W. C. Jung, J. Korean Phys. Soc., 46, 1218, (2005).

[5] W. C. Jung and K. D. Lee, J. Korean Phys. Soc, 45, 1078, (2004).

[6] R. G. Wilson, J. Appl. Phys. 54, 6879, (1983) [DOI: http://dx.doi. $\operatorname{org} / 10.1063 / 1.331993]$

[7] L. Frey, S. Bogen, L. Gong, W. Jung, and H. Ryssel, Nucl. Instrum. Methods Phys. Res. Sect. B 62 410, (1992) [DOI: http:// dx.doi.org/10.1016/0168-583X(92)95267-U].

[8] L. Gong, S. Bogen, L. Frey, W. Jung and H. Ryssel, Microelectron. Eng. 19, 495, (1992) [DOI: http://dx.doi.org/10.1016/01679317(92)90482-7].

[9] J. P. Biersack, Nucl. Instrum. Methods Phys. Res. B 35, 205, (1988) [DOI: http://dx.doi.org/10.1016/0168-583x(88)90272-8].

[10] T. E. Seidel, Nucl. Instrum. Methods Phys. Res. B 21, 96, (1987) [DOI: http://dx.doi.org/10.1016/0168-583x(87)90805-6].

[11] J. Lindhard, and M. Scharff, Phys., Rev., 124, 128, (1961) [DOI: http://dx.doi.org/10.1103/physRev. 124. 128]

[12] J. Lindhard, M. Scharff, and H. schiøtt, Mat., Fys., Medd., Dan., Vid, Selsk, 33, Nr. 14, (1963).

[13] H. Ryssel and I. Ruge, "Ion Implantation”, Wiley, New York, (1986) p. 125.

[14] A. F. Tasch and S. K. Banerjee, Nucl. Instrum. Methods Phys. Res. Sect. B 112, 177, (1996) [DOI: http://dx.doi.org/10.1016/0168583X(95)01246-X].

[15] J. F. Gibbons, "Ion implantation in Semiconductors, Part 1, Range Distribution Theory and Experiments", Proc. IEEE, March 56, Nr. 3, 295, (1968).

[16] W. Eckstein, "Computer simulation of Ion-Solid Interactions", Springer, Berlin, (1991) p. 40.

[17] Z. F. Ziegler, www.srim.org, (2013). 